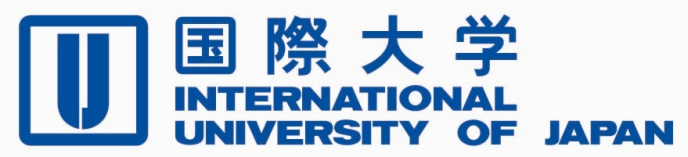

Economics \& Management Series

EMS-2011-13

\title{
Regulated Medical Fee Schedule of the Japanese Health Care System
}

\author{
Makoto Kakinaka \\ International University of Japan \\ Ryuta Ray Kato \\ International University of Japan
}

May 2011

IUJ Research Institute

International University of Japan

These working papers are preliminary research documents published by the IUJ research institute. To facilitate prompt distribution, they have not been formally reviewed and edited. They are circulated in order to stimulate discussion and critical comment and may be revised. The views and interpretations expressed in these papers are those of the author(s). It is expected that the working papers will be published in some other form. 


\title{
Regulated Medical Fee Schedule of the Japanese Health Care System*
}

\author{
Makoto Kakinaka ${ }^{\dagger} \quad$ Ryuta Ray Kato ${ }^{\ddagger}$
}

May 10, 2011

\begin{abstract}
We present a theoretical framework for investigating the effect of the Japanese government-regulated medical fee schedule, 'Shinryo-Houshu-Seido,' on the behavior of medical providers. We also discuss the optimal rule of this fee schedule for the regulator, taking into account information asymmetry between the regulator and providers. Our simple model predicts that under the current fee schedule heterogeneous providers either under-provide or over-provide medical inputs, depending on the price. Furthermore, our analytical results show that when the allocated budget is reduced to a certain level, even the second-best outcome becomes unachievable, no matter how the fee schedule is regulated. While we demonstrate that the global budget caps or the limited budget size is shown to have a clear negative effect on social welfare, we suggest that the prospect of obtaining the second-best outcome without complete information on heterogeneous providers is left to negotiation between the regulator and the budget allocator.
\end{abstract}

Keywords: asymmetric information, budget caps, regulated medical fee schedule, Japanese health care system

${ }^{*}$ We thank all participants in the Health Economics Workshop regularly held at Saku General Hospital, Nagano, Japan, and the Fifth Irvine-Japan Public Policy Conference at University of California, Irvine (March 2009) for their helpful comments and suggestions. The research fund by Promotion and Mutual Aid Corporation for Private Schools is acknowledged. Remaining errors are ours.

${ }^{\dagger}$ Graduate School of International Relations, International University of Japan, 777 Kokusai-cho, MinamiUonuma, Niigata 949-7277, Japan (email: kakinaka@iuj.ac.jp).

${ }^{\ddagger}$ Graduate School of International Relations, International University of Japan, 777 Kokusai-cho, MinamiUonuma, Niigata 949-7277, Japan (email: kato@iuj.ac.jp). 


\section{Introduction}

We present a theoretical framework for investigating the effect of 'Shinryo-Houshu-Seido' (hereafter SHS) on the behavior of medical providers. The SHS is a pricing system of medical inputs, treatments, and events regulated by the Japanese government and is also known as comprehensive fee schedule. Moreover, we discuss the optimal rule of this fee schedule for the regulator where we explicitly consider information asymmetry between the regulator and providers, and examine the effect of global budget caps on social welfare by incorporating the global budget constraint for the regulator into our framework.

As most developed countries have been struggling to reduce the ever increasing medical expenditure, Japan has also been trying to re-direct its national medical expenditure in several ways. Several drastic reforms of the public health insurance scheme have been implemented by the Japanese government over time in order to control the national medical expenditure, amongst which the revision of the SHS is one. The government has incrementally been changing the prices (fees) of many medical inputs, treatments and events covered by the public health insurance subject to the SHS. The SHS is based on a point system in which the government allocates points to all items covered by the public health insurance. Thus, all medical fees or retail prices are fully regulated by the Japanese Ministry of Health, Labor and Welfare (MHLW).

Ikegami and Campbell $(1999,2004)$ argue that government intervention through the SHS has been remarkably effective in controlling the increasing level of medical expenditure ${ }^{1}$, thus suggesting the important role played by the regulatory policy of the SHS in the Japanese health care scheme. Since point revisions are considered to induce changes in medical expenditure, it is not implausible to assume that medical providers respond to changes in the government-regulated prices of medical inputs, treatments and events that they provide their patients. Consequently, it can be argued that any point or retail price regulation of the SHS by the MHLW $^{2}$ can be considered as being strategic with the aim to control the volume of medical inputs used by medical providers. Hence, we have developed a theoretical framework to characterize the behavior of medical providers under such a fully regulated system.

We discuss the optimal choice of medical inputs by providers which differ in not only price, but also effectiveness. Upon the optimal response by medical providers to the regulated prices, we also evaluate the regulatory policy based on social welfare. In general, whether or not the regulator can achieve the first-best outcome depends on the availability of information to the regulator. In the case of the SHS in Japan, it is important for the regulator to obtain information about how providers respond to changes in regulated fees, which obviously depends on the benevolence of providers. However, since it is extremely difficult to observe the extent to which providers are interested in the welfare of their patients, any decision regarding changes in medical fees by the regulator will have to be made based on asymmetric information. The incorporation of asymmetric information on benevolence between the regulator and providers into our framework enables us to explore the second-best outcome of the SHS.

\footnotetext{
${ }^{1}$ Wagstaff (2007) summarizes the features of the Japanese health care system in comparison with other Asian countries. See also Tokita (2002), Ikegami and Campbell (1999, 2004), and Naito (2006), for details.

${ }^{2}$ In practice, the fee schedule is revised biennially at the Central Social Insurance Medical Council, which is a committee within the MHLW.
} 
In addition to information asymmetry, we also incorporate another constraint to the regulator when trying to achieve the social optimum, namely budget constraint. The budget allocated to the MHLW is usually pre-determined by the Ministry of Finance (MOF), and its size is taken as given when it regulates the SHS. In other words, the MHLW has to change regulated prices in order to fulfil its budget constraint by taking into account the optimal response of providers to achieve the social optimum with information asymmetry. We refer to the optimal rule for the regulator under the condition of limited budget constraint with asymmetric information as the third-best solution, which is indeed a condition that occurs commonly in all ministries as a result of limited budgets being pre-determined by the MOF.

We briefly present our results. First of all, the extent to which the under-provision or over-provision by heterogenous providers occurs depends on the regulated prices. If the regulated price of a new and more effective medical input is set too high compared to an old (previous) and less effective counterpart, an under-provision of the new medical input (or an over-provision of the previous one) will occur irrespective of the degree of benevolence of providers. Reversely, if the regulated price of a new medical input is too low compared to its previous counterpart, it will result in an under-provision of the previous medical input and an over-provision of the new one irrespective of the degree of benevolence of providers. More interestingly, instituting profit incentive measures among providers will bring about a better outcome when the gap of the regulated prices between a less effective (previous) and a more effective (new) medical inputs is too wide or too narrow. We can thus interpret the relationship between a new medical input and its previous counterpart as that between a generic drug and its equivalent branded with a better known label, where our current model makes a prediction that is consistent with the empirical result found by Iizuka (2007) that financial incentives among medical providers matter in the Japanese prescription drug market.

Secondly, if the regulator does not have its financial constraint, the regulator can achieve the second-best outcome by regulating prices of medical inputs when there is information asymmetry between the regulator and medical providers. The second-best outcome can be characterized by the following properties; an over- (under-) provision of a new (previous) and more (less) effective medical input by providers with profit incentives, and reversely an over(under-) provision of a previous (new) and less (more) effective medical input by providers with no profit incentives.

Finally when the allocated budget to the regulator is reduced below a certain level, even the second-best outcome becomes unachievable. We refer this situation as the third-best environment, which depends on the cost gap between a new medical input and a previous one as well as to the extent to which the allocated budget to the regulator is reduced. When the allocated budget is reduced below a certain level, we present the third-best outcome, which is characterized only by the cost gap between a new medical input and a previous one: When the cost gap is relatively small (large), an over- (under-) provision of a new medical input occurs irrespective of the degree of benevolence of providers. While we demonstrate that the global budget caps or the limited budget size is shown to have a clear negative effect on social welfare, we suggest that the prospect of obtaining the second-best outcome without complete information on heterogenous providers is left to negotiation between the regulator and the budget allocator. In Japan, the MHLW acts as the regulator, and its budget is allocated by the MOF. Thus, as long as the MHLW can negotiate with the MOF about its 
budget size, the MHLW can at least achieve the second-best outcome, even when it cannot observe benevolence of providers.

We organize our paper as follows. We first review the related literature briefly, and then present our basic framework. In Section 4, we describe the detailed models of medical providers as well as of the regulator, and then discuss the social optimal rules. In this section, we highlight the effect of budget caps as well. Finally we conclude our paper in Section 5 .

\section{The Literature}

Among many studies on the behavior of physicians as well as the reimbursement schemes of the health care system ${ }^{3}$, the argument on the choice of prescription by physicians are in particular related to our discussion. Hellerstein (1998), Coscelli (2000), Lundin (2000), and Liu, Yang, and Hsieh (2009) discuss the choice by physicians between generic drugs and its equivalent branded with a better known label, and also argue that physicians or hospitals may act as imperfect agents for their patients in the pharmaceutical market ${ }^{4}$. lizuka (2007) empirically examines the expert-client relationship in the context of the Japanese prescription drug market, and argues that the existence of markup distorts prescription decisions by physicians who often both prescribe and dispense drugs ${ }^{5}$.

While the existing literature explores the expert-client relationships such as the relationship between providers and patients, we examine the regulator-expert relationship; the interaction between the regulator and medical providers. In our model, the regulator explicitly takes into account the optimal response of medical providers when it optimally regulates the prices of medical inputs. Providers take the prices of inputs as given when they optimally choose their inputs. Providers are heterogenous in terms of their benevolence, and the regulator has no information on the benevolence of heterogenous providers when it optimally sets prices of medical inputs.

Another related literature is concerned with the effect of global budget caps. Poterba (1994) surveys the literature on global budget caps, and Van de Ven (1995) investigates the effect of a global budget in the regulated health care system. Mougeot and Naegelen (2005) studies the expenditure cap scheme in the European countries in comparison with the fee-for-service scheme by using the model in which hospitals choose the quality and the

\footnotetext{
${ }^{3}$ Many theoretical studies examine various issues related to payment systems such as prospective, and costbased payment systems (Newhouse (1983, 1996), Ellis and McGuire (1986, 1988, 1990, 1993), Dranove (1987), Pope (1989), Selden (1990), Allen and Gertler (1991), Glazer and McGuire (1994), Rogerson (1994), Ma (1994), Ma and McGuire (1997), Chalkley and Malcomson (1998), Ellis (1998), Lewis and Sappington (1999), Bos and De Fraja (2002), Beitia (2003), Boadway, Marchand, and Sato (2004), Mougeot and Naegelen (2005), Siciliani (2006), and Wright (2007)). Several studies apply mechanism-design approaches to investigate the health care system with information asymmetry (Lewis and Sappington (1999) and Beitia (2003)). See also McGuire (2000), and Dranove and Satterthwaite (2000) for the literature review.

${ }^{4}$ Grabowski and Vernon (1992), Jacobson, O'Malley, Earle, Pakes, Gaccione, and Newhouse (2006), and O'Malley, Frank, Kaddis, Rothenberg, and McNeil (2006) discuss the relationship between the prices of medical inputs and the reimbursement schemes. Ii and Ohkusa (2002) examine the price sensitivity of demand for primary care services in Japan.

${ }^{5}$ Iizuka (2007) also argues that physcians consider more finacial burdens of their patients than their own profits. Iizuka (2009) investigates the behavior of pharmaceutical firms which produce generic drugs in the regulated Japanese pharmaceutical market.
} 
cost of medical inputs, and he discusses the effectiveness of the expenditure cap policy for the reduction of medical expenditure in the European countries. While the expenditure cap policy has been implemented in order to reduce national medical expenditure in the European countries, Japan directly regulates the retail prices of medical inputs instead. The MHLW revises fees of all medical inputs covered by the public health insurance every other year in order to control the national medical expenditure. In other words, the MHLW changes all regulated prices of medical inputs in order to fulfill its budget constraint; its budget caps. We investigate the effect of budget caps on the behavior of the regulator, the MHLW, when the regulator optimally sets medical fees in order to maximize social welfare.

We now move onto our framework: Heterogenous medical providers choose medical inputs which differ in effectiveness, cost, and price (revenue), by taking prices of medical inputs as given. The regulator sets prices of medical inputs in order to maximize social welfare by considering the optimal response of medical providers without having information on the benevolence of heterogenous medical providers. The regulator also has to take into account its budget constraint when it sets the optimal prices of medical inputs. Medical providers optimally choose medical inputs by considering their patients' health as well as their own profits.

\section{The Model}

We present a theoretical framework for investigating the effect of 'Shinryo-Houshu-Seido (SHS), ' the regulated medical prices (fees), on the behavior of medical providers, by taking into account the information asymmetry between the regulator and providers. ${ }^{6}$ We only examine the relationship between the regulator and medical providers, and all aspects of the demand side such as the relationship between providers and patients are taken as given. Since we are mainly concerned with the relationship between the regulator and providers in the current Japanese public health care system, the relationship between insurers and providers is also as given.

In the context of the Japanese public health care system, the Ministry of Health, Labor and Welfare (MHLW) fully regulates prices of medical inputs covered by the public health insurance subject to SHS within its budget being predetermined by the budget allocator. The Ministry of Finance (MOF) acts as the budget allocator in Japan. This implies that the MHLW changes the regulated prices (fees) in order to satisfy its budget constraint determined by the MOF.

Furthermore, the MHLW can ex post observe economic activities of each provider in the monetary term, and the names of medical items used and the volume of them are both observable. However, the reason why each provider used a particular medical item cannot be

\footnotetext{
${ }^{6}$ We categorize hospitals and physicians as the medical provider in this paper. We do explicitly not consider the relationship between physicians and hospitals, since we believe that such a relationship is less relevant in the Japanese health care system. We do not discuss the behaviour of insurers either, since the role of insurers is very limited in the current Japanese system, as pointed out by Tokita (2002). Regarding the relationship between physicians and hospitals, see Tirole (1988) within the context of the vertical control, and also see Pauly and Redisch (1973), Shortell, Morrisey, and Conrad (1985), Custer, Moser, Musacchio, and Willike (1990), Dor and Watson (1995), Boadway, Marchand, and Sato (2004), and Crainich, Leleu, and Mauleon (2008).
} 
detected, and thus the degree of benevolence of each provider is not observable. If a medical provider is not a perfect principal to its agent (patient), then asymmetric information on the benevolence of providers between the regulator and providers does not result in the first best outcome. We explicitly consider information asymmetry in terms of the benevolence of providers between the regulator and providers, and examine the current SHS of the Japanese health care system. We also incorporate providers' freedom of choice in prescription of medical inputs which differ in effectiveness, price and cost. We can apply our model to explore the effect of the recent reform in the fee schedule in order to make providers use more generic drugs rather than brand-name ones.

We consider a society with many patients who receive medical services from providers. The number of patients is normalized at unity. In order to streamline our analysis, we simply assume that there are two medical inputs available to providers which use either of two medical inputs, $s \in\{0,1\}$ : medical input 0 is an old type with a lower cost but it is less effective, while medical input 1 is a new type with a higher cost but it is more effective. We can consider that medical input 0 and 1 correspond to generic and brand-name drugs, respectively. The cost of providing medical input $s$ is identical at $c(s)$ for all providers, such that a new medical input is more costly, i.e., $0<c(0)<c(1)$. The regulated price (fee) of medical input $s$ is set by the regulator at $p(s)$ in the SHS, the fee schedule. Note that $p(s)$ is the revenue when a provider chooses to provide its patient with medical input $s$. Given the co-payment rate $\tau \in(0,1)$, the payments to a provider from its patient and the public health insurance are $\tau p(s)$ and $(1-\tau) p(s)$, respectively. ${ }^{7}$

Each patient is heterogeneous in terms of the severity of her health condition, $\lambda$, which is distributed over $[0,1]$ according to a distribution function $F$. A patient can visit only one provider and is treated with either old or new medical input. The payoff of patient $\lambda$ who is treated with medical input $s$ is given by the health-related payoff $h(s, \lambda)$ minus her co-payment $\tau p(s)$ :

$$
w(s, \lambda)=h(s, \lambda)-\tau p(s) .
$$

In order to make our model tractable, the health-related payoff from medical input $s$ of patient $\lambda$ is given by $h(s, \lambda)=\theta-(1-s) \alpha \lambda$, where $\theta$ and $\alpha$ are both positive constants. This specification simplifies the effectiveness of a new medical input. A patient who receives a new medical input obtains the fixed health-related payoff of $\theta$ irrespective of the severity of her health condition, while the health-related payoff of a patient who receives an old medical input is $\theta-\alpha \lambda$, which decreases with a rise in the severity of her health condition. The severity of the health condition, $\lambda$, is unobservable for a patient but can be identified by a provider. As emphasized in McGuire (2000), such asymmetric information between providers and patients allows providers to act as imperfect agents due to conflicts of interests. For simplicity, we assume that providers can choose the type of medical inputs only for their own sake, so that patients always accept all medical inputs suggested by their providers. The assumption that all patients act passively could be regarded as an extreme case of the physician-induced demand studied by Evans (1974).

\footnotetext{
${ }^{7}$ The Japanese health care system has mainly been based on the fee-for-service scheme: Many medical services are reimbursed based on the fee-for-service scheme, while there have been several hospitals which moved to the prospective reimbursement scheme with the DPC (Diagnosis Procedure Combination), which is Japan's specific DRG.
} 
One crucial assumption of this study is that there are two types of providers: completely benevolent providers $(\mathrm{CBs})$ and partially benevolent providers (PBs). The fraction of CBs is $r \in(0,1)$. The type of providers is private information, and a patient visits her provider without any identification of its type. Thus, patients randomly visit either CBs or PBs, so that $r$ and $1-r$ are the shares of patients who visit $\mathrm{CB}$ and $\mathrm{PB}$, respectively.

CBs provide medical inputs only by considering their patients, while PBs provide them based not only on patients' interests but also on their own interests. That is, CBs act as perfect agents for their patients, while PBs do as imperfect agents. The payoff of CBs associated with the provision of medical input $s$ to patient $\lambda$ is simply equivalent to the payoff of patient $\lambda$ who is treated with medical input $s$ :

$$
u^{c}(s, \lambda)=w(s, \lambda) .
$$

On the other hand, the payoff of PBs associated with the provision of medical input $s$ to patient $\lambda$ also depends on their own profits (or the markup), $\pi(s)=p(s)-c(s)$, as well as the payoff of their patients $w(s, \lambda)$ :

$$
u^{p}(s, \lambda)=\rho \pi(s)+w(s, \lambda),
$$

where $\rho>0$ is the degree of a self-profit incentive that is assumed to be identical for all PBs. We assume that the degree of a self-profit incentive is not so large such that $\rho \in(0, \tau)$. Notice that for both CBs and PBs the benevolent part of the payoff, $w(s, \lambda)$, depends on patients' health-related payoff $h(s, \lambda)$ as well as their co-payments $\tau p(s)$. In our setting, providers care about the effectiveness of medical inputs they provide as well as the cost (co-payments) of medical inputs which their patients have to pay. The inclusion of patients' co-payments in the payoff of providers is consistent with the argument of Lundin (2000) that physicians take into account patients' costs significantly when they prescribe. Iizuka (2007) empirically finds that physicians consider patient's out-of-pocket costs in the prescription drug market in Japan.

Let $s^{c}(\lambda) \in\{0,1\}$ and $s^{p}(\lambda) \in\{0,1\}$ denote the choice of medical inputs to patient $\lambda$ made by $\mathrm{CBs}$ and PBs, respectively. Then we assume that social welfare is defined by the integral of the health-related payoffs of patients minus the cost incurred by the provision of medical inputs:

$$
W=r \int_{0}^{1}\left[h\left(s^{c}(\lambda), \lambda\right)-c\left(s^{c}(\lambda)\right)\right] d F(\lambda)+(1-r) \int_{0}^{1}\left[h\left(s^{p}(\lambda), \lambda\right)-c\left(s^{p}(\lambda)\right)\right] d F(\lambda) .
$$

The first term is the social welfare associated with the patients treated by CBs, while the second term corresponds to the social welfare associated with the patients treated by PBs.

We assume that the regulator can set the price of medical input $s, p(s) .{ }^{8}$ To focus on the regulated price of a newly developed medical input, we assume that the price of an old

\footnotetext{
${ }^{8}$ The fee schedule, or points allocated to all items covered by the public health insurance, are revised biennially at the Central Social Insurance Medical Council. Points set to a newly developed, or a brandname drug, are usually higher than those to an existing one, although the medical effect of a newly developed drug is similar to that of an existing one, in order to cover the research and development cost of the newly developed drug. Furthermore, points set to an existing one is usually decreased at the biennial revision of the fee schedule when a newly developed drug appears. See Ikegami and Campbell (2004) and Iizuka (2007, 2009).
} 
medical input is always set to be equal to its cost, i.e., $p(0)=c(0)$. For simplicity, we assume that the regulator controls prices to allow providers to have a profit from the provision of a newly developed medical input, but zero profit from an old medical input. We also assume that the regulator knows the ratio of each type of providers, but it cannot identify whether a provider is a $\mathrm{CB}$ or $\mathrm{PB}$, so that typical adverse selection problems occur due to information asymmetry on the type of providers between the regulator and providers. Furthermore, we assume that the regulator cannot control its own budget size $B$ as well as the co-payment rate $\tau$. $B$ and $\tau$ are predetermined outside the model. In the Japanese context, the Ministry of Health, Labor and Welfare (MHLW) is regarded as the regulator. The assumption of the pre-determined budget available to the MHLW corresponds to the concept of a global budget cap, as analyzed in Poterba (1994), Van de Ven (1995), and Mougeot and Naegelen (2005).

To analyze how regulatory policies affect providers' choice of medical inputs, we consider the following three stage circumstance: At the first stage, the regulator sets the price of a newly developed medical input, $p(1)$, by taking into account the optimal response of providers. At the second stage, providers decide which medical input is given to their patients. At the final stage, the payoffs for providers are determined.

\section{Analysis}

We first present the socially optimal outcome. The social optimality is defined by the maximized social welfare, which is given by the health-related payoffs of patients minus the cost incurred by the provision of medical inputs. Then we describe a decentralized economy, where two different types of providers optimally choose medical inputs they provide their patients, by taking the prices of medical inputs as given. This corresponds to the second stage. Furthermore, we also examine the optimal behavior of the regulator at the first stage. We assume that the regulator optimally chooses the prices of medical inputs in order to maximize social welfare in three different environments: The first environment in which the regulator optimally chooses the prices of medical inputs is that the regulator has no financial constraint with complete information on the type of providers. This environment induces the first-best outcome. The second environment is that the regulator still has no financial constraint but it has no information on the type of providers. This situation results in the second best case where there is information asymmetry in terms of the type of providers between the regulator and providers. The last environment to be considered is that the regulator has its budget constraint with information asymmetry, and the optimal outcome is called the third-best solution in this environment. In fact, the MHLW has been changing fees in the SHS in order to satisfy its pre-determined budget, and the last environment corresponds to the actual situation.

\subsection{Social Optimality}

The social optimality requires that for each patient the difference between the health-related payoff and the cost incurred by the provision of a medical input to the patient is maximized, i.e., $n(s, \lambda)=h(s, \lambda)-c(s)$ is maximized for all $\lambda$. If $n(0, \lambda)>n(1, \lambda)$ for $\lambda$, then the provision of an old type of medical inputs is optimal for $\lambda$. On the other hand, if $n(0, \lambda)<n(1, \lambda)$ for 
$\lambda$, then the provision of a new type of medical inputs is optimal for $\lambda$. Let $\kappa \equiv c(1)-c(0)>0$ denote the cost-gap between an old and a new medical inputs. We can easily prove that in the socially optimal outcome providers give all patients $\lambda<\lambda^{o}$ an old medical input, and also give all patients $\lambda>\lambda^{\circ}$ a new medical input, where

$$
\lambda^{o}=\frac{\kappa}{\alpha}
$$

This implies that a patient with the low severity of her health condition should be treated with an old and thus cheap medical input, while a patient with the high severity should be treated with a new but expensive medical input. Note that $\lambda^{o}$ determines the socially optimal critical value.

However, in general a society does not have an effective mechanism to enforce providers to choose the above defined medical inputs: Information asymmetry on the difference in benevolence among providers between the regulator and providers does not result in the above defined first best outcome. If the regulator had complete information on the difference, then the regulator would be able to differentiate prices (fees) for each provider according to its different benevolence, and the first best outcome in terms of the provision of medical inputs could be achieved. However, the benevolence of a provider is in general not observable, and the regulator could only identify the revenue of providers and medical inputs they provided their patients. Thus, the first best outcome is in general not achievable, and in the following subsection we attempt to examine a socially suboptimal outcome and discuss the second-best as well as the third-best solutions in a decentralized economy.

\subsection{Providers' Decision Problem}

We now present the optimal decision rule for providers in a decentralized economy, and then compare the derived result with the socially optimal condition. Note that providers make a decision by taking all prices as given, and their decisions are made at the second stage. Given the prices of medical inputs, $p(0)[=c(0)]$ and $p(1)$, providers choose their medical inputs in order to maximize their payoffs: CBs choose an old medical input for patient $\lambda$ if $u^{c}(0, \lambda)>u^{c}(1, \lambda)$, and a new medical input if $u^{c}(0, \lambda)<u^{c}(1, \lambda)$. PBs chooses an old medical input for patient $\lambda$ if $u^{p}(0, \lambda)>u^{p}(1, \lambda)$, and a new medical input if $u^{p}(0, \lambda)<u^{p}(1, \lambda)$.

Let denote the price difference between an old and a new medical inputs by $\delta \equiv p(1)-p(0)$. Since CBs only consider the payoffs of their patients given by (1), CBs obviously provide all patients $\lambda<\lambda^{c}$ with an old medical input and all patients $\lambda>\lambda^{c}$ with a new medical input. On the other hand, as given by (2), PBs consider not only the payoffs of their patients, but also their own profits, and we can show that PBs provide all patients $\lambda<\lambda^{p}$ with an old medical input and all patients $\lambda>\lambda^{p}$ with a new medical input such that:

$$
\lambda^{c}(\delta)=\frac{\tau \delta}{\alpha} ; \quad \lambda^{p}(\delta)=\lambda^{c}(\delta)+\frac{\rho(\kappa-\delta)}{\alpha} .
$$

Notice that $\kappa-\delta=[p(0)-c(0)]-[p(1)-c(1)]=-[p(1)-c(1)]$, where $p(1)-c(1)$ is the profit generated by the provision of a new medical input, $s=1$. If the regulator sets the

price of a new medical input to generate a positive profit, then $\kappa-\delta$ becomes negative, thus resulting in $\lambda^{p}(\delta)<\lambda^{c}(\delta)$. However, as we will see below, even when the regulated price of 
a new medical input results in a negative profit, there is still a possibility for PBs to choose to provide their patients with a new medical input due to their partial benevolence. The extent to which PBs accept a negative profit depends upon $\rho$. As expressed by (3) and (4), both of the cost gap, $\kappa \equiv c(1)-c(0)$, and the price gap, $\delta \equiv p(1)-p(0)$, between an old and a new medical inputs do matter for the critical values.

To evaluate the impact of a change in the co-payment rate, $\tau$, as well as in the price-gap between an old and a new medical inputs, $\delta$, we respectively differentiate $\lambda^{c}$ and $\lambda^{p}$ with respect to $\tau$ and $\delta$ :

$$
{\frac{\partial \lambda^{i}}{\partial \tau}}_{i=c, p} \equiv \lambda_{\tau}^{c}=\lambda_{\tau}^{p}=\frac{\delta}{\alpha}>0 ; \quad \frac{\partial \lambda^{c}}{\partial \delta} \equiv \lambda_{\delta}^{c}=\frac{\tau}{\alpha}>0 ; \quad \frac{\partial \lambda^{p}}{\partial \delta} \equiv \lambda_{\delta}^{p}=\frac{\tau-\rho}{\alpha}>0 .
$$

These imply that for both CBs and PBs the fraction of the patients treated with an old medical input increases as the co-payment rate rises. The fraction also increases when the price-gap between an old and a new medical inputs becomes wider. ${ }^{9}$

The results are quite intuitive. A rise in the co-payment rate gives a more financial burden on patients, thus letting providers which consider patients' payoffs use a cheap medical input for more patients. As long as providers consider patients' payoffs, the effect is the same, and it does not matter whether providers are completely or partially benevolent. On the other hand, the effect of an increase in the price gap between an old and a new medical inputs is slightly different, while it results in an increase in the number of patients treated with an old medical input by both CBs and PBs. Since CBs do only care about their patients' payoffs, the effect is straightforward: A wider price gap lets CBs use a cheap medical input for more patients, and the magnitude of the effect is denoted by $\frac{\tau}{\alpha}$. However, PBs consider not only their patients' payoffs but also their own financial payoffs; their profits. When the price gap becomes wider, they find it more attractive to use a more expensive medical input based on their own financial incentives. Their financial incentives weaken the first effect denoted by $\frac{\tau}{\alpha}$. The magnitude of their financial incentives is denoted by $\frac{\rho}{\alpha}$, and the magnitude of the overall effect is denoted by $\frac{\tau-\rho}{\alpha}$. As long as their financial incentives are small enough as being assumed to be $\tau-\rho>0$, an increase in the price gap between an old and a new medical inputs makes PBs to use a cheap medical input for more patients. These results suggest that the MHLW could stimulate providers to use more generic rather than brand-name drugs, by increasing the co-payment rate and/or the price of a newly developed brand-name drugs.

We now examine the relationships among the critical values. In particular, we are concerned about the relationships of two critical values, $\lambda^{c}$ and $\lambda^{p}$ with $\lambda^{o}$, the socially optimal critical value; the over-provision or the under-provision of medical inputs by each of two different providers. We can describe the four possible cases completely by different ranges of the value of the price gap, $\delta \equiv p(1)-p(0)$, and summarize the result when the prices are exogenously given to providers in the following lemma:

Lemma 1 The three critical values, $\lambda^{o}, \lambda^{c}$, and $\lambda^{p}$, satisfy that:

(1) $\lambda^{c}<\lambda^{p}<\lambda^{o}$ if $\delta<\kappa$;

(2) $\lambda^{p}<\lambda^{c}<\lambda^{o}$ if $\delta \in\left(\kappa, \frac{1}{\tau} \kappa\right)$;

(3) $\lambda^{p}<\lambda^{o}<\lambda^{c}$ if $\delta \in\left(\frac{1}{\tau} \kappa, \frac{1-\rho}{\tau-\rho} \kappa\right)$;

(4) $\lambda^{o}<\lambda^{p}<\lambda^{c}$ if $\delta>\frac{1-\rho}{\tau-\rho} \kappa$.

\footnotetext{
${ }^{9}$ The assumption of $\rho<\tau$ ensures this result in terms of the effect for partially benevolent providers.
} 
Note that $\kappa<\frac{1}{\tau} \kappa<\frac{1-\rho}{\tau-\rho} \kappa$ given the assumption of $\rho<\tau$. Note also that an increase in $\delta$ implies an increase in the price of a new medical input. (1) corresponds to the case where the regulated price of a new medical input is too low to generate a positive profit through the provision of a new medical input; $\kappa-\delta=-[p(1)-c(1)]>0$. In this case both CBs and PBs under-provide (over-provide) an old and less effective (a new and more effective) medical input in comparison with the socially optimal situation: The regulated price of a newly developed and thus more effective medical input is too low. Furthermore, CBs provide a new medical input to more patients relatively to PBs. Since PBs partially have financial incentives, the amount of the over-provision of a too cheap new medical input is relatively smaller compared to that by CBs which only care about the effectiveness of medical inputs as well as the financial burden on patients. In other words, PBs do not over-provide a new medical input as much as CBs do, due to their own financial incentives when the profit is negative.

On the other hand, if the regulated price of a new medical input is very high, then opposite case happens. (4) corresponds to this case, where both CBs and PBs over-provide (underprovide) an old and less effective (a new and more effective) medical input in comparison with the socially optimal situation: A newly developed and thus more effective medical input is too expensive. Due to their own financial incentives among PBs, the amount of the over-provision of an old medical input is relatively smaller compared to that by CBs.

(2) and (3) correspond to the cases between the two extreme cases. (2) corresponds to the case where the profit through the provision of a new medical input is positive, but the profit is relatively small $\left(\delta \in\left(\kappa, \frac{1}{\tau} \kappa\right)\right)$. In this case, similar to (1), both CBs and PBs still under-provide (over-provide) an old and less effective (a new and more effective) medical input in comparison with the socially optimal situation, and the regulated price of a new medical input is still too low, while it now guarantees to generate a positive profit. However, the relationship between the critical values of $\mathrm{PBs}$ and $\mathrm{CBs}$ is now reversed compared to (1) where the profit is negative. In case (2), the profit is positive, and PBs tend to increase the provision of a new medical input based on their own financial incentives. Such financial incentives result in more over-provision of a new medical input. (3) is the most ambiguous case, where the amount of a positive profit is larger than $\frac{1}{\tau} \kappa$ but it is not large enough over $\frac{1-\rho}{\tau-\rho} \kappa$. In this case, PBs still under-provide (over-provide) an old and less effective (a new and more effective) medical input, but CBs now over-provide (under-provide) an old and less effective (a new and more effective) medical input.

Before moving onto the regulator's rule, we should present a very interesting result: PBs always choose the critical value closer to the social optimal value compared to $\mathrm{CBs}$, when the regulated price of a new and more effective medical input is either very low or very high. This implies that the existence of financial incentives among providers results in a better outcome when the regulated price is either too low or too high.

Notice that if the regulator can obtain perfect information on the type of providers then we can use the optimal rule which can achieve the socially optimal outcome by proposing 
the different price of a new medical input to each of them according to the following rule:

$$
\begin{aligned}
& \text { CBs: } \lambda^{o}=\frac{\kappa}{\alpha}=\frac{\tau \delta}{\alpha}=\lambda^{c}(\delta), \\
& \text { PBs: } \lambda^{o}=\frac{\kappa}{\alpha}=\lambda^{c}(\delta)+\frac{\rho(\kappa-\delta)}{\alpha}=\lambda^{p}(\delta)
\end{aligned}
$$

We now examine the optimal rule of regulated prices for the regulator with asymmetric information on the type of providers. The regulator takes into account the optimal response of providers to the prices of medical inputs, and the regulator determines the regulated prices in order to maximize social welfare.

\subsection{The Regulator's Decision Problem}

We describe the first stage; the regulator's decision. The regulator determines prices of medical inputs by taking into account the optimal responses of two different types of providers. We assume that the regulator maximizes social welfare such that

$$
W(\delta)=r W^{c}(\delta)+(1-r) W^{p}(\delta)
$$

where $W^{c} \equiv \int_{0}^{\lambda^{c}}(h(0, \lambda)-c(0)) d F(\lambda)+\int_{\lambda^{c}}^{1}(h(1, \lambda)-c(1)) d F(\lambda)$ and $W^{p} \equiv \int_{0}^{\lambda^{p}}(h(0, \lambda)-$ $c(0)) d F(\lambda)+\int_{\lambda^{p}}^{1}(h(1, \lambda)-c(1)) d F(\lambda)$. $W^{c}(\delta)$ and $W^{p}(\delta)$ correspond to welfare of all patients treated by CBs and PBs, respectively, and social welfare is defined by the weighted sum of welfare. Recall that $r$ denotes the fraction of CBs.

The regulator reimburses providers for the provision of medical inputs through the SHS, and the total amount of reimbursements is given by

$$
B(\delta)=r B^{c}(\delta)+(1-r) B^{p}(\delta)
$$

where $B^{c} \equiv(1-\tau)\left[c(0)+\delta \int_{\lambda^{c}}^{1} d F(\lambda)\right]$ and $B^{p} \equiv(1-\tau)\left[c(0)+\delta \int_{\lambda^{p}}^{1} d F(\lambda)\right] . B^{c}(\delta)$ and $B^{p}(\delta)$ denote the amount of reimbursements to $\mathrm{CBs}$ and PBs, respectively. From now on, we simply assume that the severity of patients' health condition, $\lambda$, is uniformly distributed over $[0,1]$.

We now present the optimal rules for the regulator in three different environments: The first environment is that the regulator has no financial constraint with complete information on the type of providers. This environment results in the first best outcome, and we consider the first-best solution as a benchmark. The second environment is that the regulator still has no financial constraint but it has no information on the type of providers anymore. This situation corresponds to the second best case where there is information asymmetry in terms of the type of providers between the regulator and providers. The last environment to be considered is that the regulator has its budget constraint with information asymmetry. We define this case as the third-best situation, where the regulator has to satisfy its budget constraint, $\bar{B}>0$, with information asymmetry. In fact, the MHLW has been changing fees in the SHS in order to satisfy its predetermined budget every other year, and the last case corresponds to the actual situation.

In the first environment, the regulator has complete information on the type of providers, and it does not need consider its budget constraint either. In this case, the regulator sets the 
discriminated price to each provider in order to maximize (6) without considering (7), and the first-best solution, where $W^{c}(\delta)$ and $W^{p}(\delta)$ are maximized independently, is achieved. As expressed by (5a) and (5b), the regulator can attain the first best outcome by differentiating the price gap between an old and a new medical inputs for each of two different providers such that

$$
\delta^{c}=\frac{1}{\tau} \kappa ; \quad \delta^{p}=\frac{1-\rho}{\tau-\rho} \kappa .
$$

Note that the critical values of $\lambda^{o}, \lambda^{c}\left(\delta^{c}\right)$, and $\lambda^{p}\left(\delta^{p}\right)$ are identical such that $\lambda^{o}=\lambda^{c}\left(\delta^{c}\right)=$ $\lambda^{p}\left(\delta^{p}\right)$. Figure 1 illustrates the graphs of $W(\delta), W^{c}(\delta)$, and $W^{p}(\delta)$. $W^{c}(\delta)$ and $W^{p}(\delta)$ are maximized at $\delta^{c}=\frac{1}{\tau} \kappa$ and $\delta^{p}=\frac{1-\rho}{\tau-\rho} \kappa$, respectively. Notice also that $\delta^{c}<\delta^{p}$.

However, in the second environment, where the regulator has no information on the type of providers, the regulator cannot differentiate the price gap for each of two different types. In this case, the regulator has to seek the second-best solution in order to maximize (6). We still retain the assumption that the regulator does not need consider its budget constraint, so that (7) is irrelevant in this case. Then regulator optimally proposes the unique price gap to both types of providers without considering (7). Given the assumption that $\lambda$ is uniformly distributed over $[0,1]$, we can simplify the first order condition, $W^{\prime}(\delta)=0$, such that:

$$
r \hat{\lambda}_{\delta}^{c}\left(\kappa-\alpha \hat{\lambda}^{c}\right)=-(1-r) \hat{\lambda}_{\delta}^{p}\left(\kappa-\alpha \hat{\lambda}^{p}\right) .
$$

The LHS of (8) measures the marginal increment in social welfare associated with $W^{c}(\delta)$ and the RHS does with $W^{p}(\delta)$. (8) implies that the regulator has to set the unique price gap in order to equate the marginal welfare associated with $W^{c}(\delta)$ with that associated with $W^{p}(\delta)$ in the second-best environment. We manipulate (8) to obtain the second-best unique price-gap between an old and a new medical inputs, $\hat{\delta}=K \cdot[c(1)-c(0)]$, where

$$
K \equiv 1+\frac{(1-\tau)[\tau-(1-r) \rho]}{r \tau^{2}+(1-r)(\tau-\rho)^{2}}>1 .
$$

The assumption of $\rho<\tau$ ensures $K>1$. Recall that $\kappa \equiv c(1)-c(0)$, so that the regulator chooses the optimal price-gap such that $\hat{\delta}>\kappa$. Since $\kappa-\delta=[p(0)-c(0)]-[p(1)-$ $c(1)]=-[p(1)-c(1)]<0$, this implies that the regulator has to optimally set the price, $p(1)$, to induce a positive profit through the provision of a new medical input when there is information asymmetry on the type of providers between the regulator and providers. Furthermore, as illustrated in Figure 1, we can easily show

$$
\delta^{c}<\hat{\delta}<\delta^{p} .
$$

Recall that $W^{c}(\delta)$ and $W^{p}(\delta)$ are maximized at $\delta^{c}=\frac{1}{\tau} \kappa$ and $\delta^{p}=\frac{1-\rho}{\tau-\rho} \kappa$, respectively, and also that $\delta^{c}<\delta^{p}$. Since $r$ is the fraction and $0<r<1, \hat{\delta}$ which maximizes (6) should be located between $\delta^{c}$ and $\delta^{p}$. This also implies that $K \in\left(\frac{1}{\tau}, \frac{1-\rho}{\tau-\rho}\right)$.

We now discuss the optimal behavior of each of two different providers after the regulator optimally sets the price gap, $\hat{\delta}$. As we have proved in Lemma 1 , the critical values of CBs and PBs denoted by $\hat{\lambda}^{c} \equiv \lambda^{c}(\hat{\delta})$ and $\hat{\lambda}^{p} \equiv \lambda^{p}(\hat{\delta})$ depend on $\hat{\delta}$. Since $K \in\left(\frac{1}{\tau}, \frac{1-\rho}{\tau-\rho}\right)$ and $\hat{\delta}=K \cdot[c(1)-c(0)]=K \cdot \kappa$, we have $\hat{\delta} \in\left(\frac{1}{\tau} \kappa, \frac{1-\rho}{\tau-\rho} \kappa\right)$. Thus, we obtain the following result with Lemma 1 when there is information asymmetry, but the regulator does not need consider its budget constraint. 
Proposition 1 Suppose that the regulator does not have the information on the type of providers, and also that it does not have its financial constraint when it optimally sets the price gap between an old and a new medical inputs. Then completely benevolent providers (CBs) under-provide a new medical input, while partially benevolent providers (PBs) overprovide a new medical input; $\hat{\lambda}^{p}<\lambda^{o}<\hat{\lambda}^{c}$.

Note that $\delta^{c}<\hat{\delta}<\delta^{p}$. This implies that the second-best price of a new medical input is too high (low) for completely (partially) benevolent providers in comparison with their first best price of it. Thus, when the regulator optimally sets the unique price gap for both types in the second-best environment, completely benevolent providers (CBs) reduce the provision of a new medical input too much. In contrast, partially benevolent providers (PBs) provide a new medical input too much.

We now examine the last case, where the regulator faces not only information asymmetry but also its budget constraint. Denoting the total budget available to the regulator by $\bar{B}$, we now impose the constraint of $\bar{B} \geq B(\delta)$, which is also written as

$$
\bar{B} \geq(1-\tau) c(0)+(1-\tau) \delta\left\{1-r \lambda^{c}(\delta)-(1-r) \lambda^{p}(\delta)\right\} .
$$

Note that $1-r \lambda^{c}(\delta)-(1-r) \lambda^{p}(\delta)$ is the total number of patients who are treated by both types of providers with a new medical input, which is given by the property of the uniform distribution. Note also that the second term of the RHS of (10) is the total amount of reimbursements associated with the price gap between an old and a new medical inputs. Using (4), we can rewrite (10) such that

$$
\bar{B} \geq(1-\tau)\left\{c(0)+\frac{[\tau-\rho(1-r)]}{\alpha}\left[\frac{\alpha-\rho(1-r) \kappa}{\tau-\rho(1-r)}-\delta\right] \delta\right\} .
$$

Figure 2 illustrates the RHS of (11) under the assumption that (11) is binding. Note that the RHS of (11) is quadratic in terms of $\delta$. We can easily show that there is a critical value of the price gap, $\bar{\delta}$, for the RHS of (11) such that the RHS of (11) increases (decreases) as $\delta$ increases for $\delta<\bar{\delta}(\delta>\bar{\delta})$, and the critical value is given by

$$
\bar{\delta} \equiv \frac{\alpha-\rho(1-r) \kappa}{2[\tau-\rho(1-r)]} .
$$

An increase in the price gap between an old and a new medical inputs, $\delta$, consists of two effects on the total amount of reimbursements denoted by the RHS of (11): It directly increases $\delta$, and it also decreases the total number of patients who are treated by both types of providers with a new medical input. When the price gap is relatively small $(\delta<\bar{\delta})$, the former effect is larger than the latter effect, thus resulting in an increase in the total amount of reimbursements. On the other hand, the result is reversed when the price gap is large beyond $\bar{\delta}$, and the total amount of reimbursements decreases. Thus, the total amount of reimbursements, denoted by the RHS of (11), increases (decreases) as $\delta$ increases for $\delta<\bar{\delta}$ $(\delta>\bar{\delta}) \cdot{ }^{10}$

\footnotetext{
${ }^{10}$ The quadratic property also ensures the symmetric shape of the RHS of (11) around $\bar{\delta}$.
} 
The condition that (11) is binding is given by: ${ }^{11}$

$$
(1-\tau) c(0) \leq \bar{B} \leq(1-\tau)\left\{c(0)+\frac{[\alpha-\rho(1-r) \kappa]^{2}}{4 \alpha[\tau-\rho(1-r)]}\right\} .
$$

As illustrated in Figure 2, there are always two $\delta$ s at which the budget is exactly balanced when $\bar{B}$ satisfies (12). Let $\delta_{L}(\bar{B})$ and $\delta_{H}(\bar{B})$ denote the lower and the higher price-gaps, respectively, both of which exactly balance the pre-determined budget allocated to the regulator, so that $\bar{B}=B\left(\delta_{L}(\bar{B})\right)=B\left(\delta_{H}(\bar{B})\right)$ with $\delta_{L}(\bar{B})<\delta_{H}(\bar{B})$. From now on, we assume that $\bar{B}$ satisfies (12), and only examine the cases where the pre-determined budget is exactly balanced. Furthermore, we assume that the pre-determined size of the balanced budget, $\bar{B}=B\left(\delta_{L}(\bar{B})\right)=B\left(\delta_{H}(\bar{B})\right)$, is too small to let the regulator achieve the second-best outcome, so that we assume that $\delta_{L}(\bar{B})<\hat{\delta}<\delta_{H}(\bar{B}) .{ }^{12}$ In this case, the regulator has to choose either $\delta_{L}(\bar{B})$ or $\delta_{H}(\bar{B})$. We now call the optimal rule for the regulator as the third-best solution, not only when the regulator cannot choose the second-best outcome due to its budget constraint, but also when the regulator has no information on the type of providers. Then we can describe the third-best rule for the regulator as follows:

Proposition 2 The budget-constrained regulator optimally chooses the lower level of the price-gap, $\delta_{L}(\bar{B})$, if $\kappa<M$, while it optimally chooses the higher level of the price-gap, $\delta_{H}(\bar{B})$, if $\kappa>M$, where $M$ is the critical value for the cost-gap, $\kappa$, such that:

$$
M \equiv \frac{\alpha}{\rho(1-r)+[\tau-\rho(1-r)] K}
$$

In terms of the relationship between $\hat{\delta}$, the second-best price gap, and $\bar{\delta}$ which results in the highest reimbursements, there are two possibilities: Figure 3 shows the case where $\hat{\delta}<\bar{\delta}$, while Figure 4 shows the case where $\hat{\delta}>\bar{\delta}$. Since $\hat{\delta}=K \cdot \kappa$ and $\bar{\delta} \equiv \frac{\alpha-\rho(1-r) \kappa}{2[\tau-\rho(1-r)]}$, we can rewrite the condition of $\hat{\delta}<(>) \bar{\delta}$ equivalently by $\kappa<(>) M$. Due to the quadratic property of $B(\delta)$, we can show that $\delta_{L}$ is closer to $\hat{\delta}$ if $\kappa<M$ (Figure 3), while $\delta_{H}$ is closer to $\hat{\delta}$ if $\kappa>M$ (Figure 4). Thus, we can conclude that the regulator optimally chooses a smaller price gap, $\delta_{L}(\bar{B})$ (a larger price gap, $\delta_{H}(\bar{B})$ ) when $\kappa=c(1)-c(0)$, the cost gap between an old and a new medical inputs, is relatively smaller $(\kappa<M)$ (larger $(\kappa>M))$. Note that in the third-best outcome the regulator cannot choose the second-best price gap, $\hat{\delta}$, due to its budget constraint. Instead the regulator optimally chooses the price gap which is relatively closer to the second-best price gap, thus resulting in the highest social welfare when the regulator has to fulfill its budget constraint with no information on the benevolence of providers.

We now explore the optimal behaviors of two different types of providers when the regulator optimally chooses the price gap subject to its budget constraint. Each type of providers takes the unique price gap as given, which has already been set optimally by the regulator. Notice that by Proposition $2 \delta_{L}(\bar{B})<\hat{\delta}<\bar{\delta}<\delta_{H}(\bar{B})$ if $\kappa<M$, and also that

\footnotetext{
${ }^{11}$ We also impose the condition of $(1-\tau) c(0) \leq \bar{B}$. This condition ensures that the pre-determined budget, $\bar{B}$, is large enough to cover the total amount of reimbursements when both types of providers provide all their patients with an old and thus less expensive medical input.

${ }^{12}$ As illustrated in Figure 2, the regulator can always choose the second-best price gap, $\hat{\delta}$, when $\bar{B}>B(\bar{\delta})$.
} 
$\delta_{L}(\bar{B})<\bar{\delta}<\hat{\delta}<\delta_{H}(\bar{B})$ if $\kappa>M$. Let $\tilde{\lambda}^{c} \equiv \lambda^{c}(\tilde{\delta})$ and $\tilde{\lambda}^{p} \equiv \lambda^{p}(\tilde{\delta})$ denote the critical values for CBs and PBs, respectively, when the unique price gap is set on the third-best rule denoted by $\tilde{\delta} \in\left\{\delta_{L}(\bar{B}), \delta_{H}(\bar{B})\right\}$. Then, from Lemma 1, we can obtain the following lemma.

Lemma 2 Let $B_{L} \equiv B\left(\delta^{c}\right)$ and $B_{H} \equiv B\left(\delta^{p}\right)$.

(1) If $\kappa<M$, then $\delta_{L}<\delta^{c}<\hat{\delta}$ for any $\bar{B}<B_{L}$, and $\delta^{c}<\delta_{L}<\hat{\delta}$ for any $\bar{B}>B_{L}$.

(2) If $\kappa>M$, then $\hat{\delta}<\delta^{p}<\delta_{H}$ for any $\bar{B}<B_{H}$, and $\hat{\delta}<\delta_{H}<\delta^{p}$ for any $\bar{B}>B_{H}$.

Figures 5 and 6 illustrate the case of (1) of Lemma 2, depending on the values of $\bar{B}$ and $B_{L}$. Figures 7 and 8 illustrate the case of (2) of Lemma 2, depending on the values of $\bar{B}$ and $B_{H}$. In the case of (1) of Lemma 2, we have $\kappa<M$, and the third-best price gap is given at $\tilde{\delta}=\delta_{L}(<\hat{\delta})$ by Proposition 2. Since we have $\hat{\delta} \in\left(\delta^{c}, \delta^{p}\right)$ as expressed by $(9), \delta_{L}$ must satisfy either $\delta_{L}<\delta^{c}$ or $\delta_{L} \in\left(\delta^{c}, \delta^{p}\right)$, depending on the size of $\bar{B}$. Figure 5 and 6 illustrate the cases when $\delta_{L}<\delta^{c}$ and $\delta_{L} \in\left(\delta^{c}, \delta^{p}\right)$, respectively. As shown in both figures, $\delta_{L}<\delta^{c}$ and $\delta_{L} \in\left(\delta^{c}, \delta^{p}\right)$ imply $\bar{B}<B_{L}$ and $\bar{B}>B_{L}$, respectively. On the other hand, in the case of (2) of Lemma $2, \delta_{H}$ must satisfy either $\delta_{H} \in\left(\delta^{c}, \delta^{p}\right)$ or $\delta_{H}>\delta^{p}$, depending on the size of $\bar{B}$. Figure 7 and 8 illustrate the cases when $\delta_{H}>\delta^{p}$ and $\delta_{H} \in\left(\delta^{c}, \delta^{p}\right)$, respectively. Note that $\delta_{H}>\delta^{p}$ and $\delta_{H} \in\left(\delta^{c}, \delta^{p}\right)$ imply $\bar{B}<B_{H}$ and $\bar{B}>B_{H}$, respectively. We have four possible cases when the regulator optimally sets the price gap at the third-best level, depending on the budget size, $\bar{B}$, as well as the cost gap, $\kappa$. Figure 5 and 6 highlight the effect of the difference in the budget size when the cost gap is relatively small, while Figure 7 and 8 illustrate the same effect when the cost gap is relatively large.

With Lemma 1 and Lemma 2, we characterize the optimal behaviors of two different types of providers in the third-best environment in the following proposition:

Proposition 3 Suppose that the cost-gap is relatively small $(\kappa<M)$, so that the budgetconstrained regulator optimally sets the price-gap at $\tilde{\delta}=\delta_{L}$. Then,

(1) if $\bar{B}<B_{L}$, CBs and PBs both over-provide a new medical input $\left(\tilde{\lambda}^{p}<\tilde{\lambda}^{c}<\lambda^{o}\right)$, and

(2) if $\bar{B}>B_{L}$, CBs under-provide a new medical input, while PBs over-provide a new medical input $\left(\tilde{\lambda}^{p}<\lambda^{o}<\tilde{\lambda}^{c}\right)$.

On the other hand, suppose that the cost-gap is relatively large $(\kappa>M)$, so that the budget-constrained regulator optimally sets the price-gap at $\tilde{\delta}=\delta_{H}$. Then,

(3) if $\bar{B}<B_{H}$, CBs and PBs both under-provide a new medical input $\left(\lambda^{o}<\tilde{\lambda}^{p}<\tilde{\lambda}^{c}\right)$, and

(4) if $\bar{B}>B_{H}$, CBs under-provide a new medical input, while PBs over-provide a new medical input $\left(\tilde{\lambda}^{p}<\lambda^{o}<\tilde{\lambda}^{c}\right)$.

The comparison of Proposition 3 with Proposition 1 highlights the effect of the budget constraint for the regulator on the optimal behaviors of providers. Proposition 1 corresponds to the case where the regulator sets the second-best price gap without its budget constraint, while Proposition 3 does to the case where the regulator sets the third-best price gap with its budget constraint. As has been proved in Proposition 1, when the regulator sets the secondbest price gap without considering its limited budget size, we always have the case in which 
completely benevolent providers (CBs) under-provide a new medical input, while partially benevolent providers (PBs) over-provide it $\left(\hat{\lambda}^{p}<\lambda^{o}<\hat{\lambda}^{c}\right)$. On the other hand, when the regulator is subject to its limited budget constraint and thus it can only set the third-best price gap, it becomes more complicated, depending on the size of its limited budget as well as of the cost gap between an old and a new medical inputs. As shown in Proposition 3, when the regulator has to fulfill its budget constraint but its limited size is relatively large $\left(\bar{B}>B_{L}\right.$ or $\left.\bar{B}>B_{H}\right)$, then the limited budget constraint has no effect irrespective of the size of the cost gap, $\kappa$, and we still have $\hat{\lambda}^{p}<\lambda^{o}<\hat{\lambda}^{c}$; the under-provision of a new medical input by CBs, but the over-provision of it by PBs. However, when the limited size of the budget constraint is small enough $\left(\bar{B}<B_{L}\right.$ or $\left.\bar{B}<B_{H}\right)$, the under- or over-provision by both types occurs, depending on the size of the cost gap, $\kappa$. When the cost gap between an old and an new medical inputs is relatively small $(\kappa<M)$, the over-provision of a new medical input by both types occurs, while the under-provision of it by both types occurs when the cost gap is relatively large $(\kappa>M)$.

In the context of the Japanese health care system, the Ministry of Health, Labor and Welfare (MHLW) behaves as a regulator, and it completely regulates all the prices of medical inputs covered by the public health insurance. Medical inputs include drugs, medical treatments, medical procedures, and medical events. The MHLW obviously have to limit its activities within its allocated budget, and it cannot ignore its limited budget constraint when it changes prices (fees) of medical inputs for nation's welfare every other year. Thus, in practice, the third-best situation is most likely to occur in the Japanese health care system.

Furthermore, it is often argued that more advanced medical treatments are more laborintensive, and this implies that more advanced and thus more effective medical inputs tend to be more costly to provide patients. If this is true, it is more likely to have the case of $\kappa>M$. In addition, the size of the budget allocated to the MHLW is determined by the Ministry of Finance (MOF), and the MOF might make the budget of the MHLW more tight in order to reduce the huge amount of government deficits ${ }^{13}$. In fact, the new political power tends to reduce the total amount of the government budget, and the resources available to the public health insurance tends to decrease. This implies that the condition of $\bar{B}<B_{H}$ and $\kappa>M$ is more likely to be given to the supply side of the Japanese health care system through the SHS, and the under-provision of a new medical input by both types would occur in the near future. If the SHS induces the under-provision of a new medical input, then it would also affect the Japanese pharmaceutical industry. As Kurata (2009) points out, there might be a positive correlation between the existing SHS and the profitability of the Japanese pharmaceutical industry. Kurata (2009) argues that regulated high prices of brand name drugs have induced strong incentives among the Japanese pharmaceutical industry to invest in the development of new and competitive drugs over the world. The reduced budget of the MHLW would also generate disincentives among the Japanese pharmaceutical industry for the development of competitive drugs.

We notice that there are two sources to distort the first-best economy; information asymmetry between the regulator and providers, and the too-tight budget constraint for the

\footnotetext{
${ }^{13}$ Ihori, Kato, Kawade, and Bessho (2009) evaluate the reforms of the Japanese public health insurance started in year 2006 in the dynamic general equilibrium framework with overlapping generations, where the effect of government deficits is explicitly considered.
} 
regulator. In reality, it seems very difficult to present remedies for the first problem, namely information asymmetry, in the actual health care system. However, it would be easier to cope with the other problem; the budget size of the MHLW. The MHLW might strategically be able to negotiate with the MOF to expand its budget size for achieving the second-best outcome. As long as the MHLW can expand its budget size to $\bar{B}=B(\hat{\delta})$, or the MOF simply allocates the budget to the MHLW up to $\bar{B}=B(\hat{\delta})$, the MHLW can at least achieve the second-best outcome by changing prices in the SHS, even if it cannot observe the benevolence of heterogenous providers.

\section{Concluding Remarks}

We have developed a theoretical framework to explore the effect of 'Shinryo-Houshu-Seido (SHS),' the fully regulated fee schedule of the Japanese health care system, on the behavior of medical providers, and have also discussed the optimal policy rule for the regulator where we have explicitly considered information asymmetry between the regulator and providers. We have also incorporated the global budget constraint for the regulator into our framework, and have examined the effect of global budget caps on social welfare.

We present our results here. First of all, the extent to which the under-provision or over-provision by heterogenous providers occurs depends upon the regulated prices. If the regulated price of a new and thus more effective medical input is relatively too high than that of an old and less effective medical input, then the under-provision of the new medical input (the over-provision of the old medical input) occurs irrespective of the degree of benevolence of providers. On the other hand, if the regulated price of a new medical input is relatively too low than that of an old medical input, then the completely reverse situation occurs; the under-provision of the old medical input and the over-provision of the new medical input irrespective of the degree of benevolence of providers. More interestingly, a profit incentive among providers results in a better outcome when the gap of the regulated prices between an old and a new medical inputs is too wide or too narrow. Our prediction with the model is consistent with the empirical result of Iizuka (2007) that financial incentives among medical providers matter in the Japanese prescription drug market.

Secondly, the regulator can only achieve the second-best outcome by regulating the prices of medical inputs due to information asymmetry between the regulator and medical providers, if the regulator does not have its financial constraint when it regulates the prices (fees) of medical inputs. In the second-best situation, the under-provision of an old medical input (the over-provision of a new medical input) by providers with profit incentives always occurs, while the over-provision of an old medical input (the under-provision of a new medical input) by purely benevolent providers always occurs.

Finally, if the regulator faces its tight budget constraint, then the regulator cannot achieve the second-best outcome either. We refer this situation to the third-best environment, in which the results depend upon the cost gap between an old and a new medical inputs as well as the degree of tightness of its limited budget. When the limited budget size is small less than a critical value, then the results only depend upon the cost gap between an old and a new medical inputs which medical providers have to bear: When the cost gap is relatively small, the over-provision of a new medical input occurs, while the under-provision of it 
occurs when the cost gap is relatively large. This result does not depend upon the degree of benevolence of medical providers. Due to the tight budget allocated to the regulator, the regulator can at most achieve the third-best outcome, and this implies that budget caps induces a welfare loss when the regulator tries to optimally regulate prices (fees) of medical inputs with asymmetric information. In the Japanese context, the MHLW acts as the regulator, and its budget is pre-determined by the MOF. Thus, this result also suggests that the MHLW might strategically be able to negotiate with the MOF to expand its budget size for achieving the second-best outcome. As long as the MHLW can expand its budget size beyond the critical value, or the MOF simply allocates the enough budget to the MHLW, then the MHLW can at least achieve the second-best outcome by changing fees in the SHS, even if it cannot observe the benevolence of heterogenous providers.

We should notice that there are two sources to distort the first-best economy in the actual SHS; information asymmetry between the MHLW and providers, and the too-tight budget constraint for the MHLW. In reality, it seems very difficult to present remedies for the first problem, namely information asymmetry, in the actual health care system. However, it would be easier to cope with the other problem; the budget size of the MHLW, and the cooperation between the MHLW and the MOF would be able to achieve the second-best outcome.

\section{References}

Allen, R., And P. Gertler (1991): "Regulation and the Provision of Quality to heterogeneous Consumers: The Case of Prospective Pricing of Medical Services," Journal of Regulatory Economics, 3, 361-375.

Beitia, A. (2003): "Hospital Quality Choice and Market Structure in a Regulated Duopoly," Journal of Health Economics, 22, 1011-1036.

Boadway, R., M. Marchand, and M. Sato (2004): "An Optimal Contract Approach to Hospital Financing," Journal of Health Economics, 23, 85-110.

Bos, D., And G. De Fraja (2002): "Quality and Outside Capacity in the Provision of Health Services," Journal of Public Economics, 84, 199-218.

Chalkley, M., and J. M. Malcomson (1998): "Contracting for Health Services when Patient Demand does not Reflect Quality," Journal of Health Economics, 17, 1-19.

Coscelli, A. (2000): "The Importance of Doctors' and Patients' Preferences in the Prescription Decision," Journal of Industrial Economics, 48, 349-369.

Crainich, D., H. Leleu, and A. Mauleon (2008): "The Optimality of Hospital Financing System: The Role of Physician-Manager Interactions," International Journal of Health Care Finance and Economics, 8, 245-256.

Custer, W. S., J. W. Moser, R. A. Musacchio, and R. J. Willike (1990): "The Production of Health Care Services and Changing Hospital Reimbursement: The Role of Hospital-Medical Staff Relationships," Journal of Industrial Economics, 9, 167-192. 
Dor, A., And H. Watson (1995): "The Hospital-Physician Interaction in U.S. Hospitals: Evolving Payment Schemes and their Incentives," European Economic Review, 39, 795802.

Dranove, D. (1987): "Rate-Setting by Diagnosis Related Groups and Hospital Specilization," RAND Journal of Economics, 18, 417-427.

Dranove, D., And M. A. SAtTerthwaite (2000): "The Industrial Organization of Health Care Markets," in Handbook of Health Economics, vol. 1B, ed. by A. J. Culyer, and J. P. Newhouse. North Holland, Amsterdam.

Ellis, R. P. (1998): "Creaming, Skimping and Dumping: Provider Competition on the Intensive and Extensive Margins," Journal of Health Economics, 17, 537-555.

Ellis, R. P., And T. G. McGuire (1986): "Provider Behaviour under Prospective Reimbursement," Journal of Health Economics, 5, 129-151.

(1988): "Insurance Principles and the Design of Prospective Payment Systems," Journal of Health Economics, 7, 215-237.

(1990): "Optimal Payment Systems for Health Services," Journal of Health Economics, 9, 375-396.

(1993): "Supply-Side and Demand-Side Cost Sharing in Health Care," Journal of Economic Perspectives, 7, 135-151.

Evans, R. G. (1974): "Supplier-Induced Demand: Some Empirical Evidence and Implications," in The Economics of Health and Medical Care, ed. by M. Perlman. Macmillan, London.

Glazer, J., And T. G. McGuire (1994): "Payer Competition and Cost Shifting in Health Care," Journal of Economics and Management Strategy, 3(1), 71-92.

Grabowski, H. G., and J. M. Vernon (1992): "Brand Royalty, Entry and Price Competition in Pharmaceuticals after the 1984 Drug Reform Act," Journal of Law and Economics, 35, 331-350.

Hellerstein, J. K. (1998): "The Importance of the Physician in the Generic versus TradeName Prescription Decision," RAND Journal of Economics, 29, 108-136.

Ihori, T., R. R. Kato, M. Kawade, and S. Bessho (2009): "The Reform of the Public Health Insurance and Economic Growth of Japan," GSIR Working Papers: Economic Analysis and Policy Series EAP09-6, International University of Japan.

II, M., And Y. Ohkusa (2002): "Price Sensitivity of the Demand for Medical Services for Minor Ailments: Econometric Estimates Using Information on Illnesses and Symptoms," Japanese Economic Review, 53, 154-166.

IrzukA, T. (2007): "Expert's Agency Problems: Evidence from the Prescription Drug Market in Japan," RAND Journal of Economics, 38, 844-862. 
- (2009): "Generic Entry in a Regulated Pharmaceutical Market," Japanese Economic Review, 60, 63-81.

Ikegami, N., And J. C. Campbell (1999): "Health Care Reform in Japan: The Virtues of Muddling Through," Health Affairs, 18, 56-75.

(2004): "Japan's Health Care System: Containing Costs and Attempting Reform," Health Affairs, 23, 26-36.

Jacobson, M., A. J. O’Malley, C. C. Earle, J. Pakes, P. Gaccione, and J. P. Newhouse (2006): "Does Reimbursement Influence Chemotherapy Treatment For Cancer Patients?," Health Affairs, 25, 437-443.

Kurata, K. (2009): "The Development of New Drugs in the Japanese Pharmaceutical Industry (in Japanese)," RIETI Highlight, 28, 10-13.

Lewis, T., And D. Sappington (1999): "Using Subjective Risk Adjusting to Prevent Patient Dumping in the Health Care Industry," Journal of Economics and Management Strategy, 8, 351-382.

LiU, Y.-M., Y.-H. K. YAng, And C.-R. Hsieh (2009): "Financial Incentives and Physicians' Prescription Decisions on the Choice between Brand-Name and Generic Drugs: Evidence from Taiwan," Journal of Health Economics, 28, 341-349.

Lundin, D. (2000): "Moral Hazard in Physician Prescription Behavior," Journal of Health Economics, 19, 632-662.

MA, C.-T. A. (1994): "Health Care Payment Systems: Cost and Quality Incentives," Journal of Economics and Management Strategy, 3, 93-112.

Ma, C.-T. A., And T. G. McGuire (1997): "Optimal Health Insurance and Provider Payment," American Economic Review, 87(4), 685-704.

McGuire, T. (2000): "Physician Agency," in Handbook of Health Economics, vol. 1A, ed. by A. J. Culyer, and J. P. Newhouse. North Holland, Amsterdam.

Mougeot, M., and F. Naegelen (2005): "Hospital Price Regulation and Expenditure Cap Policy," Journal of Health Economics, 24, 55-72.

Naito, H. (2006): "The Japanese Health-Care System and Reimbursement for Dialysis," Peritoneal Dialysis International, 26, 155-161.

Newhouse, J. P. (1983): "Two Prospective Difficulties with Prospective Payment of Hospitals, or it's Better to be a Resident than a Patient with a Complex Problem," Journal of Health Economics, 2, 269-274.

(1996): "Reimbursing Health Plans and Health Providers: Efficiency in Production versus Selection," Journal of Economic Literature, 34, 1236-1263. 
O’Malley, A. J., R. G. Frank, A. Kaddis, B. M. Rothenberg, and B. J. McNeil (2006): "Impact of Alternative Interventions on Changes in Generic Dispensing Rates," Health Services Research, 41, 1876-1894.

Pauly, M. V., and M. Redisch (1973): "The Not-for-profit Hospital as a Physicians' Cooperative," American Economic Review, 63, 87-99.

Pope, G. C. (1989): "Hospital Nonprice Competion and Medicare Reimbursement Policy," Journal of Health Economics, 8, 147-172.

Poterba, J. (1994): "A Skeptic's View of Global Budget Caps," Journal of Economic Perspectives, 8, 67-73.

Rogerson, W. P. (1994): "Choice of Treatment Intensities by a Non-Profit Hospital under Prospective Pricing," Journal of Economics and Management Strategy, 3, 7-51.

Selden, T. M. (1990): "A Model of Capitation," Journal of Health Economics, 9, 397-409.

Shortell, S., M. A. Morrisey, and D. A. Conrad (1985): "Economic Regulation and Hospital Behavior: The Effects on Medical Staff Organization and Hospital-Physician Relationships," Health Services Research, 20, 597-628.

Siciliani, L. (2006): "Selection of Treatment under Prospective Payment Systems in the Hospital Sector," Journal of Health Economics, 25, 479-499.

Tirole, J. (1988): The Theory of Industrial Organization. The MIT Press, Cambridge, MA.

Tokita, T. (2002): "The Prospects for Reform of the Japanese Healthcare System," Pharmacoeconomics, 20, 55-66.

VAn De Ven, W. P. M. M. (1995): "Regulated Competition in Health Care: With or Without a Global Budget?," European Economic Review, 39, 786-794.

Wagstaff, A. (2007): "Health Systems in East Asia: What can Developing Countries Learn from Japan and the Asian Tigers?," Health Economics, 16, 441-456.

Wright, D. J. (2007): "Specialist Payment Schemes and Patient Selection in Private and Public Hospitals," Journal of Health Economics, 26, 1014-1026. 
Figure 1

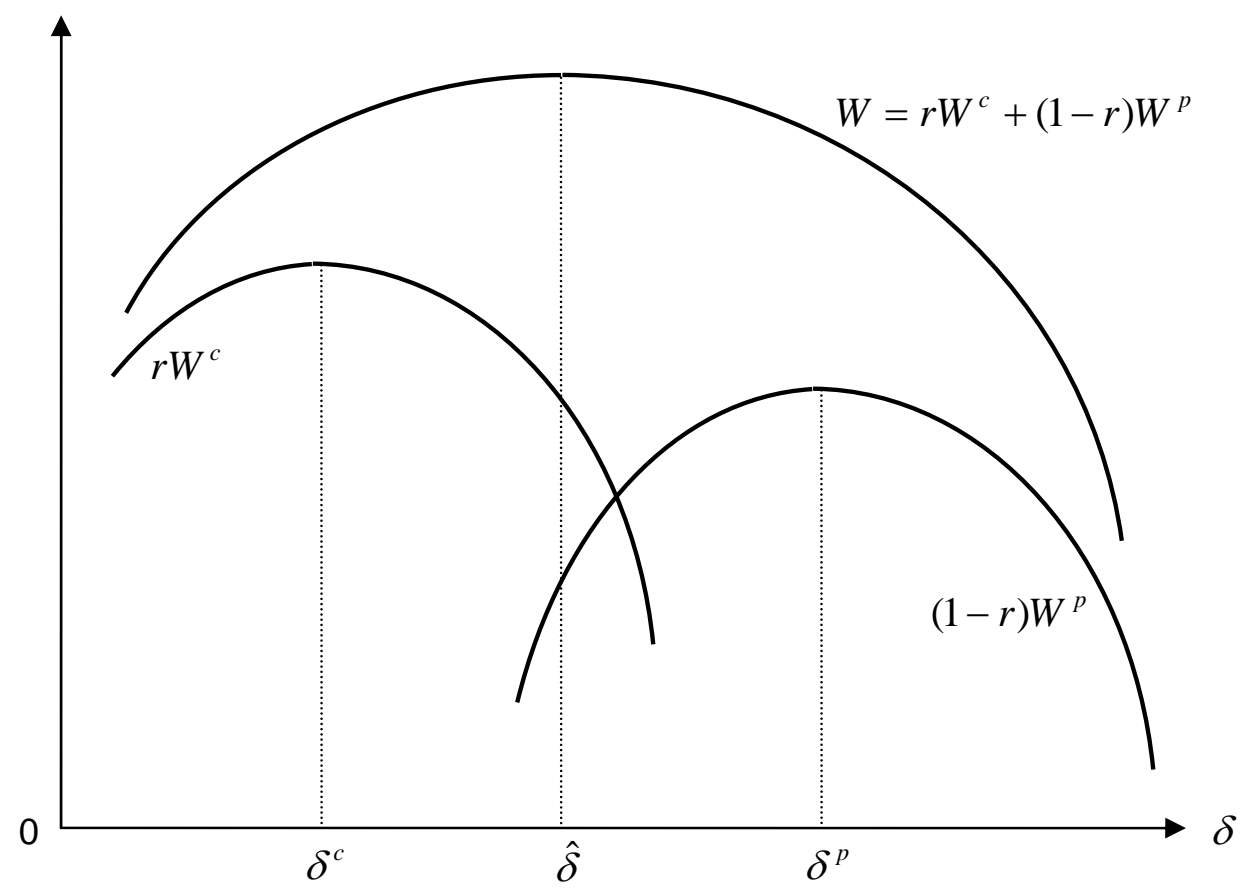

Figure 2

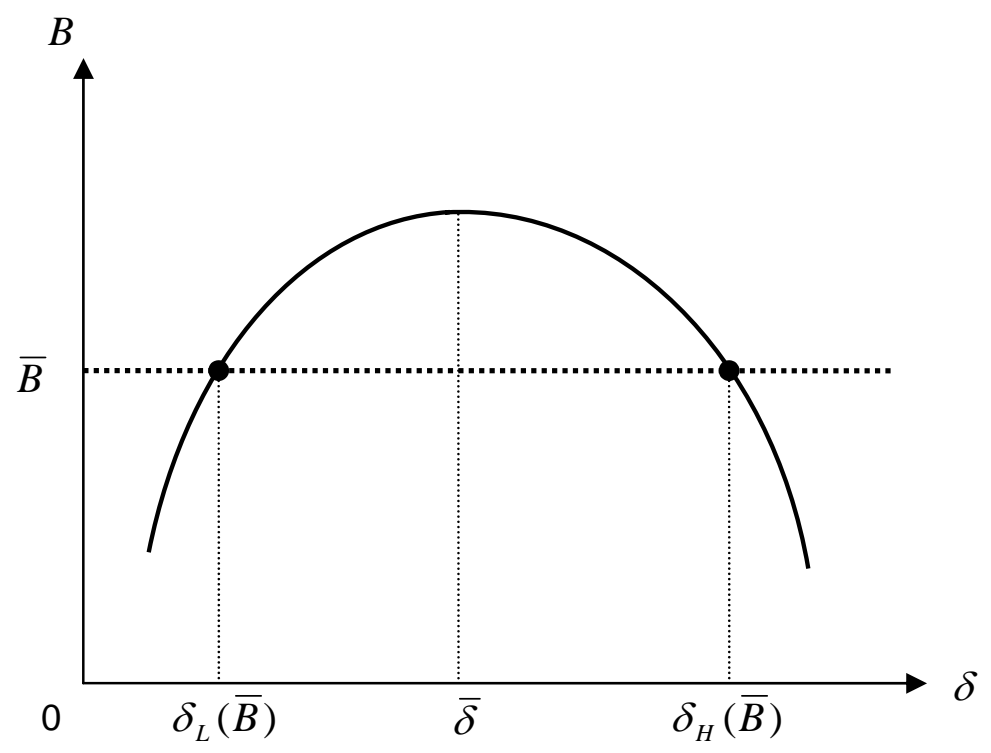


Figure 3

Case I: $\hat{\delta}<\bar{\delta}$

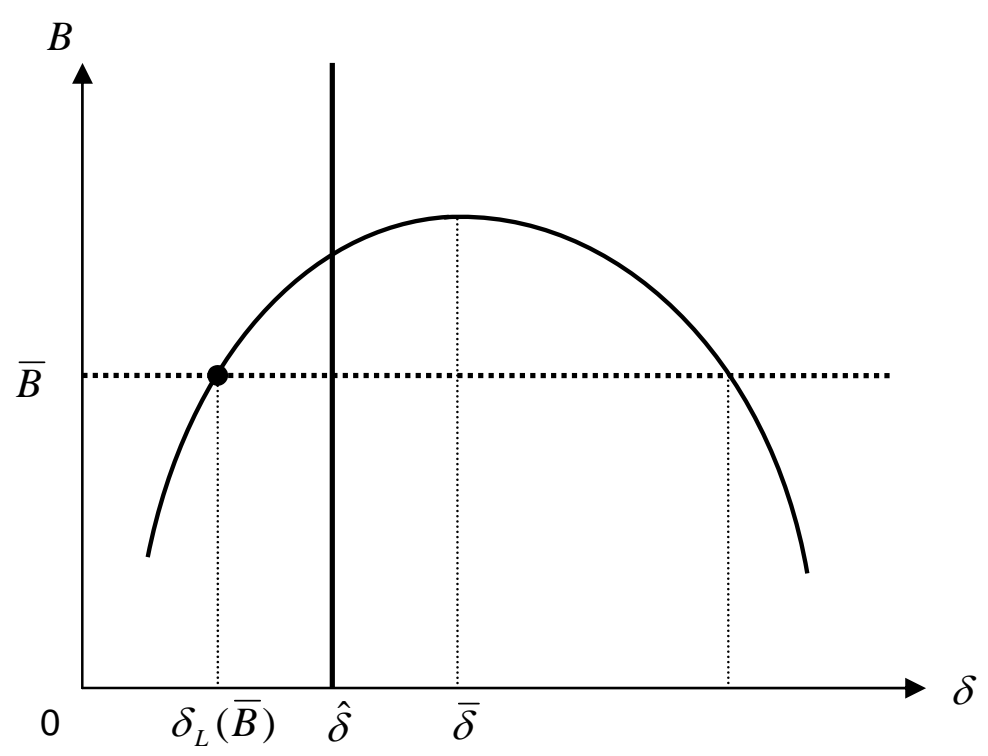

Figure 4

Case II: $\hat{\delta}>\bar{\delta}$

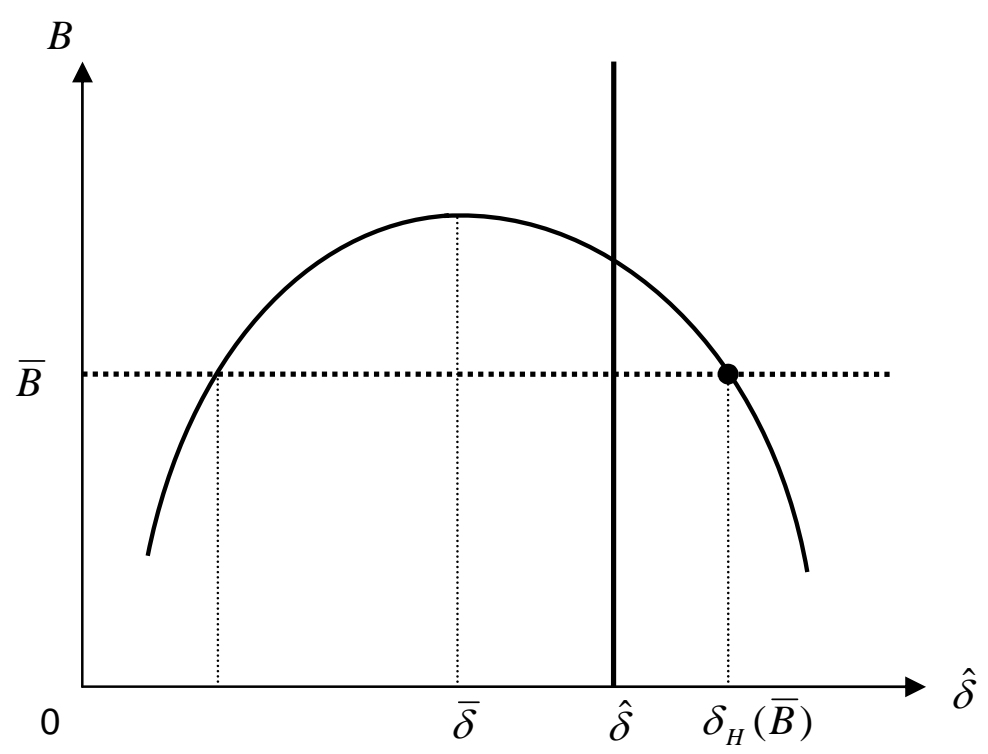


Figure 5

Case I: $\hat{\delta}<\bar{\delta}$ and $\bar{B}<B_{L}$

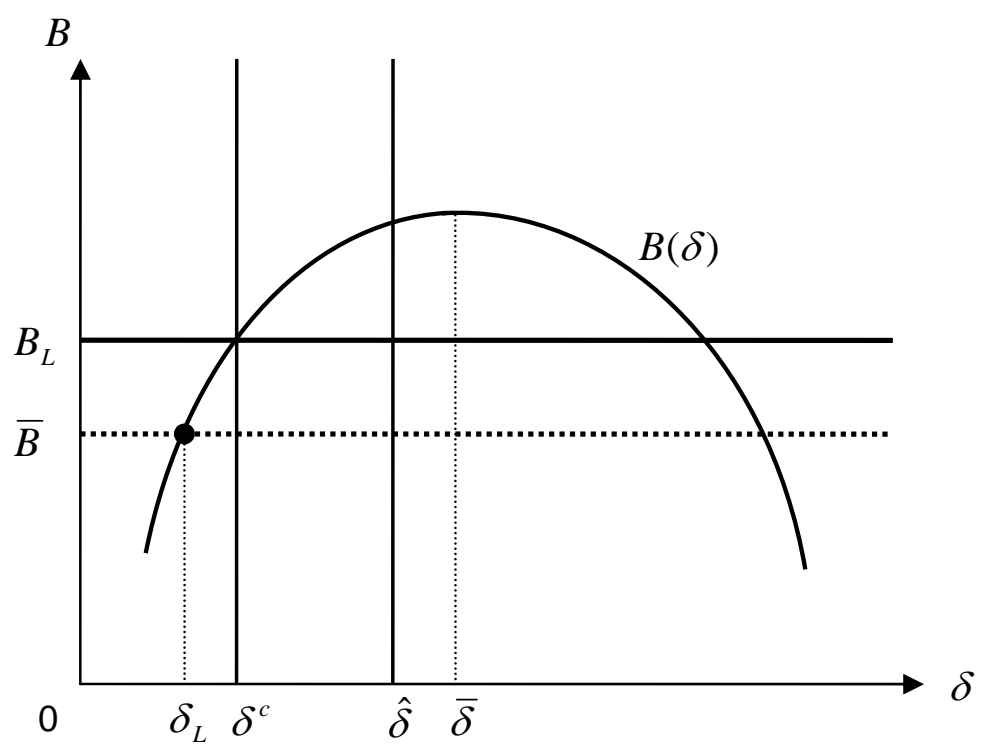

Figure 6

Case II: $\hat{\delta}<\bar{\delta}$ and $\bar{B}>B_{L}$

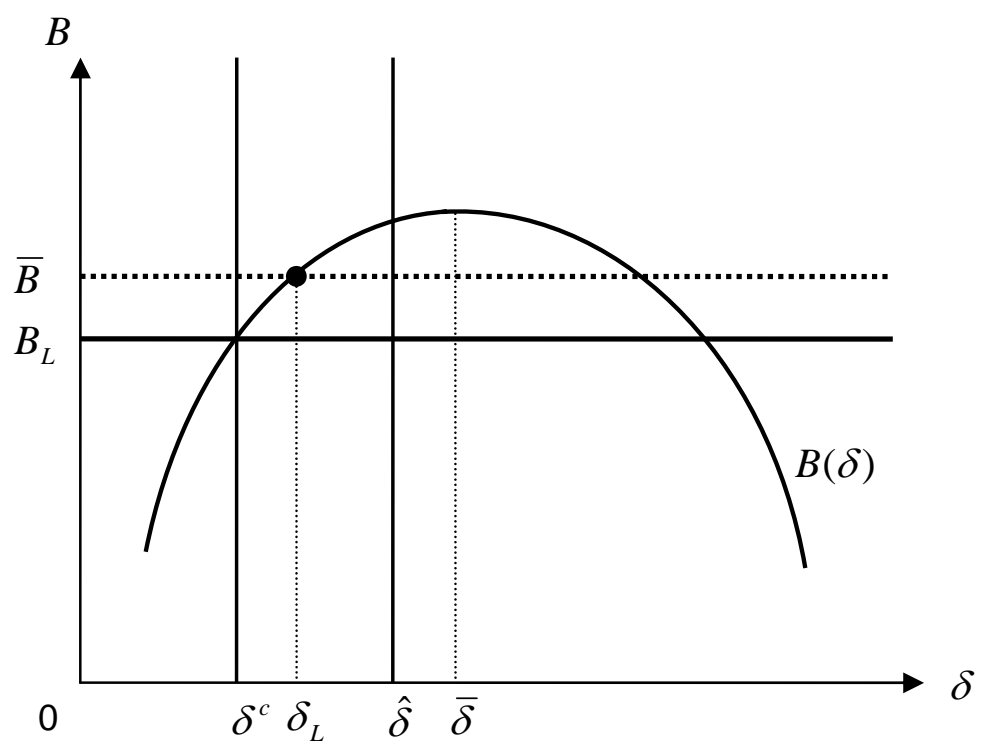


Figure 7

Case III: $\hat{\delta}>\bar{\delta}$ and $\bar{B}<B_{H}$

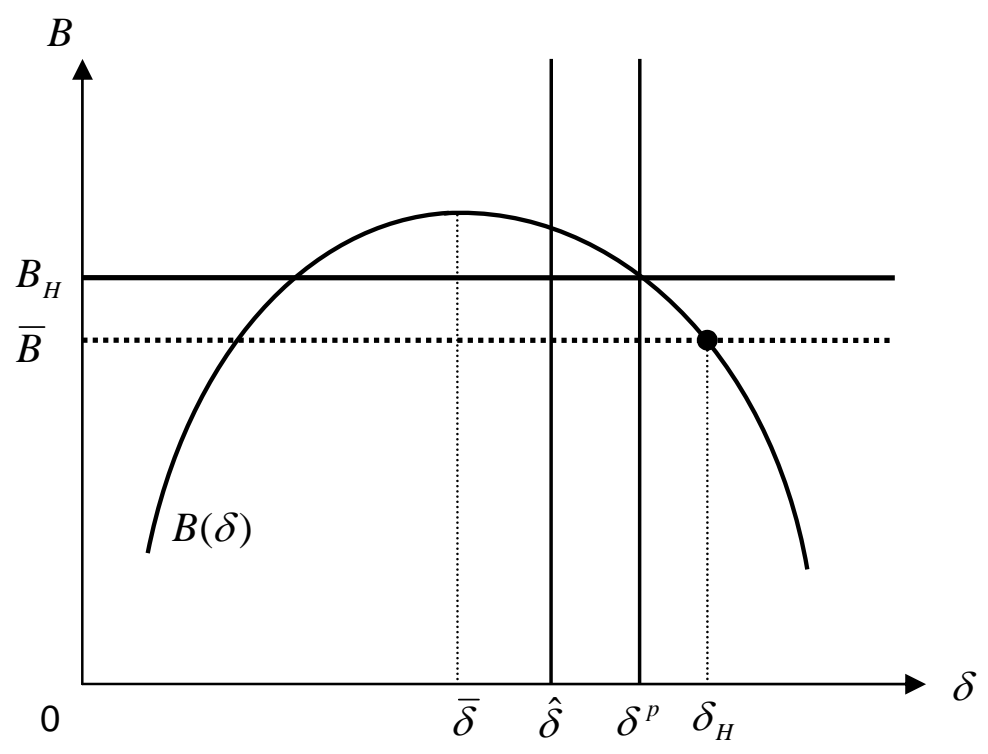

Figure 8

Case IV: $\hat{\delta}>\bar{\delta}$ and $\bar{B}>B_{H}$

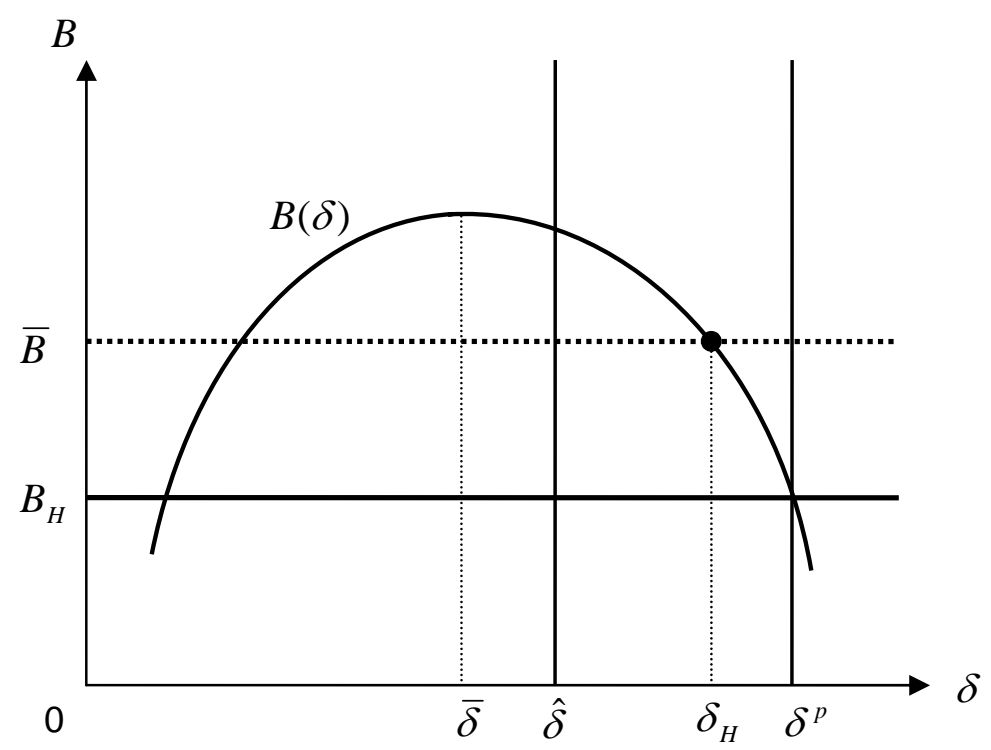

